\title{
Anisotropy of the quark anti-quark potential in a magnetic field
}

\author{
Claudio Bonati \\ Dipartimento di Fisica dell'Università di Pisa and INFN - Sezione di Pisa, \\ Largo Pontecorvo 3, I-56127 Pisa, Italy \\ E-mail: bonatiddf.unipi.it

\section{Massimo D’Elia} \\ Dipartimento di Fisica dell'Università di Pisa and INFN - Sezione di Pisa, \\ Largo Pontecorvo 3, I-56127 Pisa, Italy \\ E-mail: delia@df.unipi.it
}

\section{Marco Mariti}

Dipartimento di Fisica dell'Università di Pisa and INFN - Sezione di Pisa, Largo Pontecorvo 3, I-56127 Pisa, Italy

E-mail: mariti@df.unipi.it

\section{Michele Mesiti}

Dipartimento di Fisica dell'Università di Pisa and INFN - Sezione di Pisa, Largo Pontecorvo 3, I-56127 Pisa, Italy

E-mail: michele.mesiti@df.unipi.it

\section{Francesco Negro*}

Dipartimento di Fisica dell'Università di Pisa and INFN - Sezione di Pisa,

Largo Pontecorvo 3, I-56127 Pisa, Italy

E-mail: fnegro@pi.infn.it

\section{Francesco Sanfilippo}

School of Physics and Astronomy, University of Southampton, SO17 1BJ Southampton, United Kindgdom

E-mail: f.sanfilippoesoton.ac.uk

We study how the potential of a static quark anti-quark pair for $N_{f}=2+1 \mathrm{QCD}$ at the physical point gets modified by the presence of a constant and uniform magnetic field, $e \vec{B}$. We observe an anisotropy in the potential, which gets steeper in the directions transverse to $\vec{B}$ than in the longitudinal one. By comparing to the case at zero magnetic field, we show that the string tension increases (decreases) in the perpendicular (parallel) direction as a function of $|e \vec{B}|$, while the Sommer parameter and the absolute value of the Coulomb coupling display the opposite behavior.

The 32nd International Symposium on Lattice Field Theory

23-28 June, 2014

Columbia University New York, NY

${ }^{*}$ Speaker. 


\section{Introduction and Motivations}

In the recent past, the study of the possible effects of an intense mangetic field on strong interactions has significantly increased [1]. These studies are motivated by the need for a better understanding of the several phenomenological contexts in which large $B$ fields are actually present: from the physics of compact astrophysical objects, to that of non-central heavy ion collisions (HIC) [2] and of the early Universe. In these contexts the magnetic fields are estimated to span from $10^{10}$ Tesla up to $10^{16}$ Tesla $\left(|e| B \sim 1 \mathrm{GeV}^{2}\right)$.

Under such extreme conditions, gluon fields, even though not directly coupled to electromagnetic fields, may be subjected to significant modifications, through effective QED-QCD interactions, mediated by quark loop effects. This possibility has been confirmed by lattice QCD simulations $[3,4,5,6,7]$, resulting also in unexpected behaviors, like inverse magnetic catalysis $[4,7,8]$.

A relevant attribute of strong interactions is the confining potential: in the heavy quark limit, it is related to the expectation value of Wilson loops. Hence, it emerges as a property of gauge fields only. Then, it is interesting to ask though a magnetic background can have an influence on the static quark-antiquark potential. Indeed, many studies have considered the possibility for the potential to become anisotropic $[9,10,11,12]$, a fact that may have relevant phenomenological implications, like a modification of the bound states of heavy quarks.

On the lattice, anisotropies in the gauge field distributions due to the magnetic field have already been observed. In particular, in $[5,6]$ the average plaquettes taken in different planes have been shown to be different.

Here, we discuss an exploratory study about the dependence of the static potential on the magnetic field, based on numerical simulations of $N_{f}=2+1$ QCD at the physical point, discretized by stout improved staggered quarks and the tree-level improved Symanzik gauge action. We compute the expectation values of Wilson loops in the presence of a magnetic background, and determine from them the static potential for quark-antiquark separations perpendicular or parallel to the $B$ field. We perform simulations at zero temperature, adopting magnetic fields up to $|e| B \sim 1 \mathrm{GeV}^{2}$ and three different lattice spacings. Our results clearly reveal the emergence of anisotropies in the potential [13]. By fitting it with the Cornell parameterization, we show that both the string tension and the Coulomb coefficient depends on $B$ and are anisotropic.

\section{Simulation details}

We consider QCD with $N_{f}=2+1$ quark flavors in the presence of an external magnetic field, with isospin symmetry broken only by the electric charges. The external electromagnetic field enters the QCD action through the quark covariant derivatives $D_{\mu}=\partial_{\mu}+i g A_{\mu}^{a} T^{a}+i q_{f} A_{\mu}$, where $q_{f}$ is the electric charge of the quark and $A_{\mu}$ is the abelian gauge four-potential. On the lattice, $S U(3)$ covariant derivatives are built in terms of the link variables $U_{i ; \mu}$ ( $i$ labels the position and $\mu$ the direction); the introduction of the electromagnetic field amounts to adding also proper [13] $U(1)$ phases, $U_{i ; \mu} \rightarrow u_{i ; \mu} U_{i ; \mu}$, such that they produce the desired external field. In the present work, we choose the external field to be uniform and oriented along the $\hat{z}$ axis. 
We discretize the theory on a lattice adopting improved actions both in the gauge and in the fermionic sector of the theory. We write the Euclidean partition function as:

$$
\mathscr{Z}(B)=\int \mathscr{D} U e^{-\mathscr{S}_{Y M}[U]} \prod_{f=u, d, s} \operatorname{det}\left(D_{\mathrm{st}}^{f}[U, B]\right)^{1 / 4},
$$

where $\mathscr{D} U$ is the functional integration over the $S U(3)$ link variables; we keep the $U(1)$ phases fixed through the simulations. $\mathscr{S}_{Y M}$ is the tree level improved Symanzik action [14] and it involves the real part of the trace of $1 \times 1$ and $1 \times 2$ planar loops and $D_{\mathrm{st}}^{f}$ is the staggered Dirac operator, built up in terms of two times stout-smeared links [15].

We performed simulations at the physical value of the pion mass, $m_{\pi} \sim 135 \mathrm{MeV}$, at three different values of the lattice spacing $a=0.2173 \mathrm{fm}, 0.1535 \mathrm{fm}, 0.1249 \mathrm{fm}$. A symmetric lattice, of fixed physical volume $V=(a L)^{4} \simeq(5 \mathrm{fm})^{4}$, was adopted, with $L=24,32,40$ respectively for the three lattice spacings. We tuned the bare parameters according to the line of constant physics reported in [16], choosing $\beta=3.55,3.67,3.75$ from the coarsest to the finest lattice spacing.

Sampling has been done employing the Rational Hybrid Monte-Carlo (RHMC) algorithm, with statistics around $10^{3}-10^{4}$ molecular dynamics (MD) time units for each value of $|e| B$, for $e \vec{B}$ oriented along the $\hat{z}$ axis, going up to $|e| B \sim 1 \mathrm{GeV}^{2}$.

We extract the spin-averaged potential of a static $\bar{Q} Q$ pair, separated by a distance $\vec{R}$, from the large time behavior of the average rectangular Wilson loop $W(\vec{R}, T)$, where $T$ is its time extension. Usually one can average over all the three possible orientations of $\vec{R}$; in our case, since rotation symmetry is partially broken, we computed separately the averages over different directions. This gives us the possibility to check whether $V(\vec{R})$ is a central potential or not. We obtain the potential as

$$
a V(a \vec{n})=\lim _{n_{t} \rightarrow \infty} \log \left(\frac{\left\langle W\left(a \vec{n}, a n_{t}\right)\right\rangle}{\left\langle W\left(a \vec{n}, a\left(n_{t}+1\right)\right)\right\rangle}\right),
$$

where $\vec{n}$ and $n_{t}$ denote the spatial and temporal sides of the loop in lattice units. Basically, we plot the right-hand side of 2.2 as a function of $n_{t}$ and we search for a stable plateau at large times; in this region of $n_{t}$ we extract the potential by performing a fit to a constant function. In this computation, we restrict ourselves to the cases of $\vec{n}$ normal or parallel to $e \vec{B}$.

The UV noise which affects the observable in Eq. 2.2 can be reduced by using a proper smearing procedure. Specifically, we performed one step of HYP smearing [17] for temporal links, using the smearing parameters corresponding to the HYP2 action [18], and $N_{S M}$ steps of APEsmearing [19] for spatial links, with $\alpha_{\mathrm{APE}}=0.25$.

Since APE-smearing treats symmetrically all the spatial directions, one could expect it to wash out possible anisotropies. Thus, we studied how the results depends on the number of smearing steps $\left(N_{S M}\right)$ and, after having checked that they do not significantly depend on $N_{S M}$ (see next section), we chose $N_{S M}=24$. We determined the statistical errors on the observable defined in Eq. (2.2) and those on the parameters of the fitted plateau by performing a bootstrap analysis, in order to take correlations into account.

\section{Numerical Results}

First of all we report, in the left panel of Fig. 1, an example of the logarithm of Wilson loop ratios, defined in Eq. (2.2). We plot the combination as a function of $n_{t}$ for different APE-smearing 

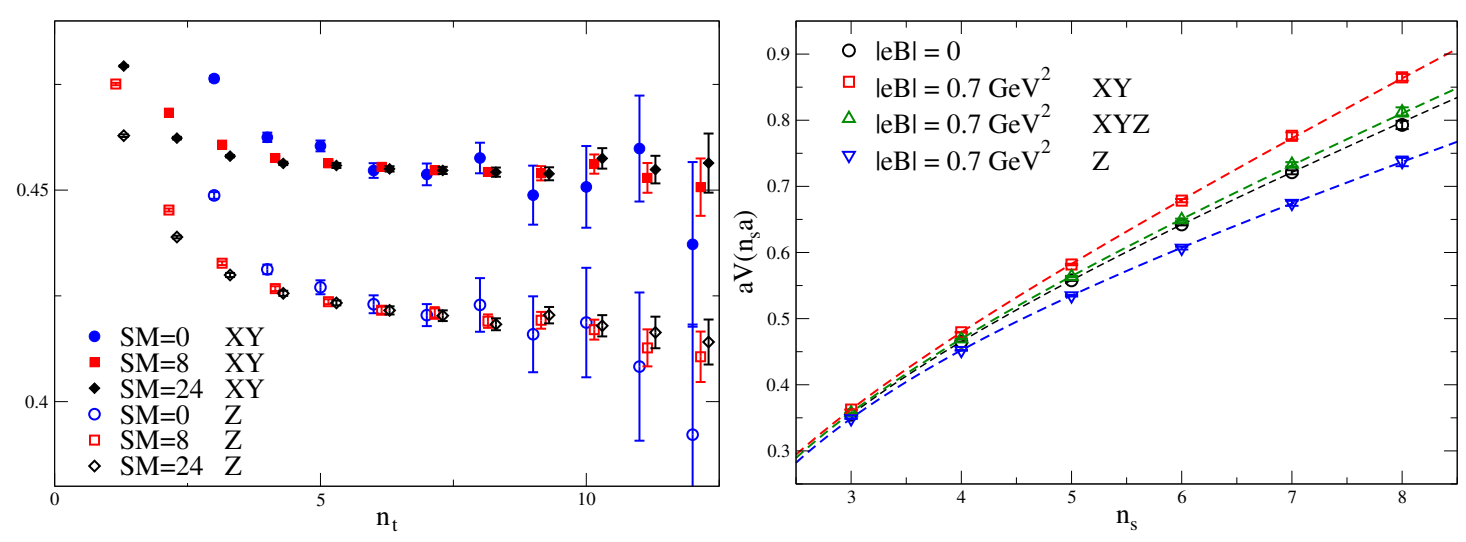

Figure 1: Left. Wilson loop combination defined in the r.h.s. Eq. (2.2) for $|\vec{n}|=3$ as a function of $n_{t}$. We plot results after several values of the APE smearing level. Data are from the simulations on the $32^{4}$ lattice at $|e| B=0.97 \mathrm{GeV}^{2}$. Right. $Q \bar{Q}$ potential both for $|e| B=0$ and for $e B=0.7 \mathrm{GeV}^{2}$ on the finest $40^{4}$ lattice.

levels. Data have been obtained at spatial distance $|\vec{n}|=3$ and for $|e| B=0.97 \mathrm{GeV}^{2}$ on the $32^{4}$ lattice. We show results averaged separately over the longitudinal $(Z)$ or transverse $(X Y)$ directions. In both cases we can see a well defined plateau; moreover, we observe that anisotropies clearly appear. It is evident that smearing has no perceptible effect on such anisotropies, so we can safely adopt a number of smearing levels which gives a good noise/signal ratio.

In the right panel of Fig. 1 we plot the static $Q \bar{Q}$ potential, as a function of the quark separation, determined for our smallest lattice spacing $a=0.1249 \mathrm{fm}$. We plot the results obtained for $|e| B \simeq$ $0.7 \mathrm{GeV}^{2}$ together with those for $|e| B=0$ for comparison (in this case we averaged over the spatial directions). For $|e| B \neq 0$ we see an anisotropic behavior, with a remarkable separation between the values of the potential along the $Z$ or $X Y$ directions. By comparing with the $|e| B=0$ case we see that $V(\vec{R})$ increases in the transverse directions and decreases in the longitudinal direction. This peculiar behavior is observed for all the explored lattice spacings and starting from fields of about $|e| B \simeq 0.2 \mathrm{GeV}^{2}$. In the same figure, we show the potential obtained by averaging Wilson loops over the three directions (denoted by $X Y Z$ ) also for the $|e| B \neq 0$ case. Interestingly, we notice that such an average produces a potential which is very close to that computed for $|e| B=0$; hence, the influence of $|e| B$ on the static potential is almost completely hidden.

To characterize the dependence of the potential on the external field, we fitted it, for each value of $B$ and separately for transverse and longitudinal directions, with the Cornell parametrization:

$$
a V\left(\text { and } \hat{d}=\hat{c}_{d}+\hat{\sigma}_{d} n+\frac{\alpha_{d}}{n},\right.
$$

where $\hat{d}$ is the unit vector along the $z$ or $x y$ directions, $\alpha_{d}$ the Coulomb coupling, $\hat{\sigma}_{d}$ is the string tension, and $\hat{c}_{d}$ a constant term; we use the label $d$ to take into account the dependence on the direction. A non-trivial result is that Eq. 3.1 fits reasonably well the measured potentials for all the explored fields, with $\chi^{2} /$ d.o.f. $\lesssim 1$, in a distance range going from $\sim 0.3$ to $\sim 1 \mathrm{fm}$. This means that we can interpret the modification of the potential simply as a modification of the Cornell fit parameters. A bootstrap analysis has been performed to determine the statistical errors of the fit parameters. 

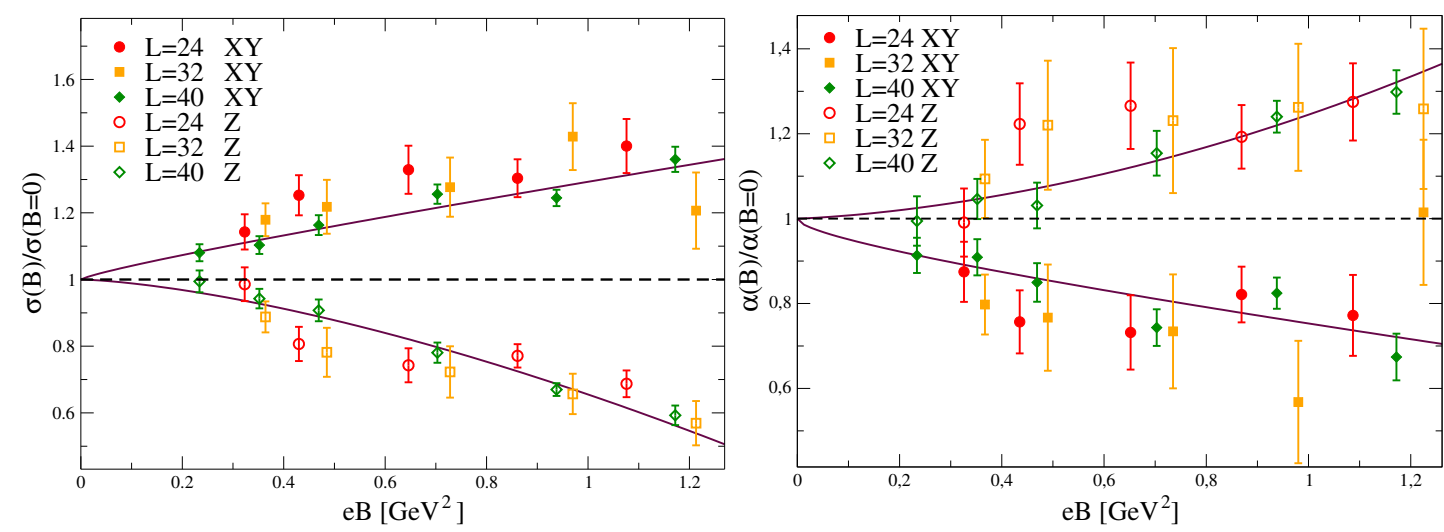

Figure 2: The ratios $R^{\sigma}$ (Left) and $R^{\alpha}$ (Right) along the $Z$ and $X Y$ directions. The solid lines are best fits according to Eq. (3.3), for the $L=40$ data.

To better study how the fitted parameters depend on $|e| B$, we normalize them according to the values they take for $|e| B=0$ and at the same lattice spacing: we compute the ratios

$$
R^{\mathscr{O}_{d}}(|e| B)=\frac{\mathscr{O}_{d}(|e| B)}{\mathscr{O}(|e| B=0)}
$$

and plot them in Figs. 2 Left, 2 Right and 3 respectively for $\mathscr{O}_{d}=\hat{\sigma}_{d}, \alpha_{d}$ and $\hat{r}_{0 d}$, where $r_{0}$ is the Sommer parameter which can be computed solving the equation $r_{0}^{2} \sigma=1.65+\alpha$. These quantities display an anisotropy which derives from that of the potential; indeed, in the corresponding ratio, we observe significant splittings which are of the order of $10-20 \%$ for the largest values of $|e| B$. In particular, the string tension increases (decreases), as a function of $|e| B$, in the trasverse (longitudinal) direction, while both the Coulomb coupling and the Sommer parameter show an opposite behavior.

Our results depend mildly on the lattice spacing, however with our present accuracy we cannot perform a proper continuum extrapolation. To give an idea of the kind of dependence of the ratios of Eq. 3.2 on the external field, we fit the $L=40$ data (i.e. those at the smallest lattice spacing) according to the following power-law ansatz:

$$
R^{\mathscr{O}_{d}}(|e| B)=1+A^{\mathscr{O}_{d}}(|e| B)^{C^{\mathscr{O}_{d}}}
$$

the results of the best fit are shown as solid lines in Figures 2 and 3 and the corresponding fitting parameters are reported in Table 1.

\section{Conclusions}

In this work, we discussed a new effect on the QCD vacuum induced by an intense $e \vec{B}$-field. Qualitatively, the physical origin of the observed anisotropy is related to the effective couplings between the photon and the gluon degrees of freedom, which arise from quark loop effects. A clear explanation is, anyhow, still missing. At the perturbative level [6], the effective action predicts an increase of the chromoelectric field components orthogonal to $e \vec{B}$ (see also Ref. [11]), and a suppression of the longitudinal one; this is in agreement with the observed anisotropies at the 
plaquette level $[5,6]$. Since confinement is related to the formation of a chromoelectric flux-tube, this result suggests an increase (decrease) of the string tension in the direction trasverse (parallel) to $e \vec{B}$, as we have found. Possible anisotropies in the static potential have been predicted also in Ref. [9], in particular a decrease of the Coulomb coupling in the transverse direction, which is consistent with our observations. A similar scenario has been observed by adopting an holographic approach [20].

This study is exploratory and claims for extensions in various directions. Here, we have limited ourselves to the computation of the potential in the $X Y$ and $Z$ directions; in future studies it will be interesting to investigate the full angular dependence. Moreover, an investigation of the profile of the flux tube along the various directions could give a clearer picture regarding the influence of $|e| B$ on the chromoelectric fields between static color charges.

Another direction for future investigations is related to the phenomenological consequences of our results. It is important to notice that, in order to have predictions valid also for the physics non-central heavy ion collisions, it will be necessary to extend our study to finite temperature. In the recent past, many works have concentrated on the physics of mesons (spectrum, production and decay rates) in the presence of a strong magnetic background [21, 22]. The results obtained in these works will likely get corrections due to the presence of the anisotropies we have found. We stress that even slight changes in the energy levels may sizably modify cross sections, production and decay rates [22]. Related to this issue, it would be of great importance to perform direct lattice measurement of heavy quark bound states in the presence of a magnetic background.

\section{Acknowledgments}

FS received funding from the European Research Council under the European Communitys Seventh Framework Programme (FP7/2007-2013) ERC grant agreement No 279757. FN acknowledges financial support from the EU under project Hadron Physics 3 (Grant Agreement n.

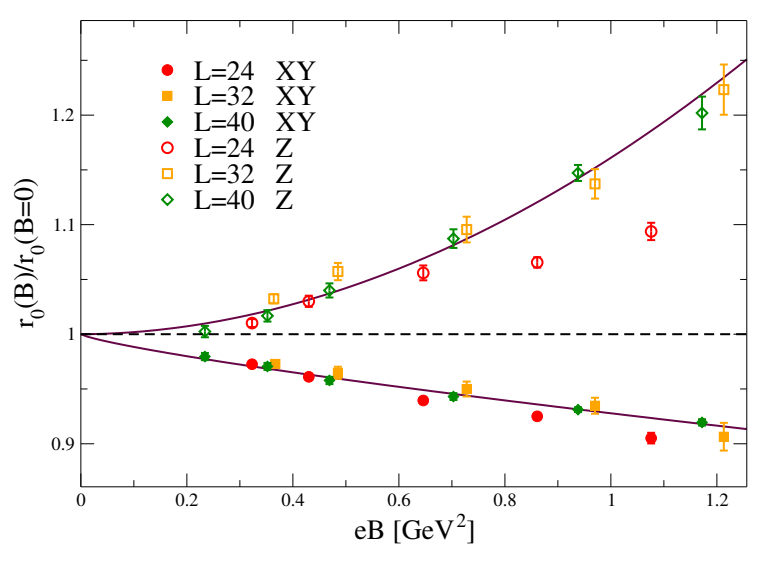

Figure 3: The ratio $R^{r_{0}}$ along the $Z$ and the $X Y$ directions. The solid line is a fit according to Eq. (3.3) for the $L=40$ data.

\begin{tabular}{|c|c|c|c|}
\hline $\mathscr{O}_{d}$ & $A^{\mathscr{O}_{d}}$ & $C^{\mathscr{O}_{d}}$ & $\chi^{2} /$ dof \\
\hline$\alpha_{X Y}$ & $-0.24(3)$ & $0.7(2)$ & 1.5 \\
\hline$\alpha_{Z}$ & $0.24(3)$ & $1.7(4)$ & 0.3 \\
\hline$\sigma_{X Y}$ & $0.29(2)$ & $0.9(1)$ & 1.1 \\
\hline$\sigma_{Z}$ & $-0.34(1)$ & $1.5(1)$ & 0.9 \\
\hline$r_{0 X Y}$ & $-0.072(2)$ & $0.79(5)$ & 0.6 \\
\hline$r_{0 Z}$ & $0.161(6)$ & $1.9(1)$ & 1.3 \\
\hline
\end{tabular}

Table 1: A summary of the results for the parameters $A$ and $C$ of Eq. (3.3). The parameters have been obtained by fitting the ratios of the various observable for our finest lattice $L=40$. 
283286). This work was partially supported by the INFN SUMA project. Simulations have been performed on the Blue Gene/Q Fermi at CINECA, based on the agreement between INFN and CINECA (under INFN projects PI12 and NPQCD).

\section{References}

[1] D. Kharzeev, K. Landsteiner, A. Schmitt and H. U. Yee Lect. Notes Phys. 8712013.

[2] V. Skokov, A. Y. Illarionov and V. Toneev Int. J. Mod. Phys. A 24, 59252009.

[3] M. D'Elia, S. Mukherjee and F. Sanfilippo Phys. Rev. D 82, 051501 2010; M. D'Elia and F. Negro, Phys. Rev. D 83, 114028 (2011); M. D’Elia, M. Mariti and F. Negro, Phys. Rev. Lett. 110, 082002 (2013); E.-M. Ilgenfritz, M. Muller-Preussker, B. Petersson and A. Schreiber, Phys. Rev. D 89, no. 5, 054512 (2014).

[4] G. S. Bali, F. Bruckmann, G. Endrodi, Z. Fodor, S. D. Katz, S. Krieg, A. Schafer and K. K. Szabo, JHEP 1202, 044 (2012).

[5] E. -M. Ilgenfritz, M. Kalinowski, M. Muller-Preussker, B. Petersson and A. Schreiber, Phys. Rev. D 85, 114504 (2012).

[6] G. S. Bali, F. Bruckmann, G. Endrodi, F. Gruber and A. Schaefer, JHEP 1304, 130 (2013).

[7] F. Bruckmann, G. Endrodi and T. G. Kovacs, JHEP 1304, 112 (2013).

[8] I. A. Shovkovy, Lect. Notes Phys. 871, 13 (2013).

[9] V. A. Miransky and I. A. Shovkovy, Phys. Rev. D 66, 045006 (2002).

[10] M. N. Chernodub, arXiv:1001.0570 [hep-ph].

[11] B. V. Galilo and S. N. Nedelko, Phys. Rev. D 84, 094017 (2011).

[12] S. Ozaki, Phys. Rev. D 89, 054022 (2014).

[13] C. Bonati, M. D'Elia, M. Mariti, M. Mesiti, F. Negro and F. Sanfilippo Phys. Rev. D 89, 1145022014.

[14] P. Weisz, Nucl. Phys. B 212, 1 (1983). G. Curci, P. Menotti and G. Paffuti, Phys. Lett. B 130, 205 (1983) [Erratum-ibid. B 135, 516 (1984)].

[15] C. Morningstar and M. J. Peardon, Phys. Rev. D 69, 054501 (2004).

[16] Y. Aoki, S. Borsanyi, S. Durr, Z. Fodor, S. D. Katz, S. Krieg and K. K. Szabo, JHEP 0906, 088 (2009); S. Borsanyi, G. Endrodi, Z. Fodor, A. Jakovac, S. D. Katz, S. Krieg, C. Ratti and K. K. Szabo, JHEP 1011, 077 (2010).

[17] A. Hasenfratz and F. Knechtli, Phys. Rev. D 64, 034504 (2001).

[18] M. Della Morte, A. Shindler and R. Sommer, JHEP 0508, 051 (2005).

[19] M. Albanese et al. [APE Collaboration], Phys. Lett. B 192 (1987) 163.

[20] R. Rougemont, R. Critelli and J. Noronha Phys. Rev. D 91, no. 6, 066001 (2015).

[21] J. Alford and M. Strickland, Phys. Rev. D 88, 105017 2013; M. Chernodub, Phys. Rev. Lett. 106, 142003 (2011); Y. Hidaka and A. Yamamoto, Phys. Rev. D 87, 094502 (2013); M. Frasca, JHEP 1311, 099 (2013); N. Callebaut, D. Dudal and H. Verschelde, JHEP 1303, 033 (2013); M. Andreichikov et al., Phys. Rev. D 87, 094029 (2013); C. Machado et al., Phys. Rev. D 89, 074027 (2014); X. Guo, S. Shi, N. Xu, Z. Xu and P. Zhuang, arXiv:1502.04407.

[22] C. S. Machado, F. S. Navarra, E. G. de Oliveira, J. Noronha and M. Strickland Phys. Rev. D 88, 034009 2013; P. Filip PoS CPOD 2013, 0352013. 